\title{
PTEN Mutation Identified in Patient Diagnosed with Simultaneous Multiple Cancers
}

\author{
Hye Sung Won, MD, PhD ${ }^{1}$ \\ Eun Deok Chang, MD, PhD² \\ Sae Jung $\mathrm{Na}$, MD, $\mathrm{PhD}{ }^{3}$ \\ In Yong Whang, $\mathrm{MD}, \mathrm{PhD}^{4}$ \\ Dong Soo Lee, MD, PhD \\ Sun Hyong You, MD, PhD ${ }^{6}$ \\ Yong Seok Kim, MD, $P h D^{6}$ \\ Jeong Soo Kim, MD, PhD
}

PTEN hamartoma tumor syndrome is a spectrum of disorders characterized by unique phenotypic features including multiple hamartomas caused by mutations of the tumor suppressor gene PTEN. Cowden syndrome and Bannayan-Riley-Ruvalcaba syndrome are representative diseases, and both have several common clinical features and differences. Because PTEN mutations are associated with an increased risk of malignancy including breast, thyroid, endometrial, and renal cancers, cancer surveillance is an important element of disease management. We report a germline mutation of the PTEN (c.723dupT, exon 7) identified in a young woman with a simultaneous occurrence of breast cancer, dermatofibrosarcoma protuberans, and follicular neoplasm. This case suggests that it is critical for clinicians to recognize the phenotypic features associated with these syndromes to accurately diagnose them and provide preventive care.
Departments of ${ }^{1}$ Internal Medicine, ${ }^{2}$ Hospital Pathology, ${ }^{3}$ Nuclear Medicine,

${ }^{4}$ Radiology, ${ }^{5}$ Radiation Oncology, and

${ }^{6}$ Surgery, Uijeongbu St. Mary's Hospital, College of Medicine, The Catholic University of Korea, Uijeongbu, Korea

\section{Key words}

Hamartoma syndrome, Breast neoplasms, Dermatofibrosarcoma protuberans
Correspondence: Jeong Soo Kim MD, PhD Department of Surgery, Uijeongbu St. Mary's Hospital, College of Medicine, The Catholic University of Korea, 271 Cheonbo-ro,

Uijeongbu 11765, Korea

Tel: 82-31-820-3048

Fax: 82-31-847-2127

E-mail: drbreast@catholic.ac.kr

Received December 6, 2017

Accepted February 27, 2018

Published Online February 27, 2018

\section{Introduction}

Phosphatase and tensin homolog (PTEN) hamartoma tumor syndrome (PHTS) includes four major clinically distinct syndromes related to germline mutations in the tumor suppressor PTEN: Cowden syndrome (CS), Bannayan-RileyRuvalcaba syndrome (BRRS), Proteus syndrome (PS), and Proteus-like syndrome (PSL) [1]. These diseases are characterized by unregulated cellular proliferation leading to the formation of numerous hamartomas in various organs [1]. The diagnosis of PHTS is confirmed by the identification of a germline mutation in PTEN along with their unique clinical characteristics.

CS is the most well-known PHTS, which is characterized by multiple disorganized benign growths or hamartomas with mucocutaneous manifestations as a pathognomonic feature. It is rarely identified before adulthood, and has shown an increased risk of both benign and malignant tumors [1,2]. BRRS is a congenital disorder known to be diagnosed early 
in life, unlike CS. Clinical characteristics of BRRS include macrocephaly, lipomas, and hamartomatous intestinal polyposis, and in males, pigmented macules of the glans penis $[1,2]$. It is of note that an increased risk of malignancy has not been formally documented in BRRS [1,3]. Both diseases have respectively distinctive phenotypic features and they also share significant clinical overlaps.
We report the case of a young woman diagnosed with breast cancer, dermatofibrosarcoma protuberans, and follicular neoplasm who had the pathogenic PTEN mutation. Ethical Committee approval was obtained from the Institutional Review Board of The Catholic University of Korea (No. UC17ZESE0138), and an informed consent was provided.
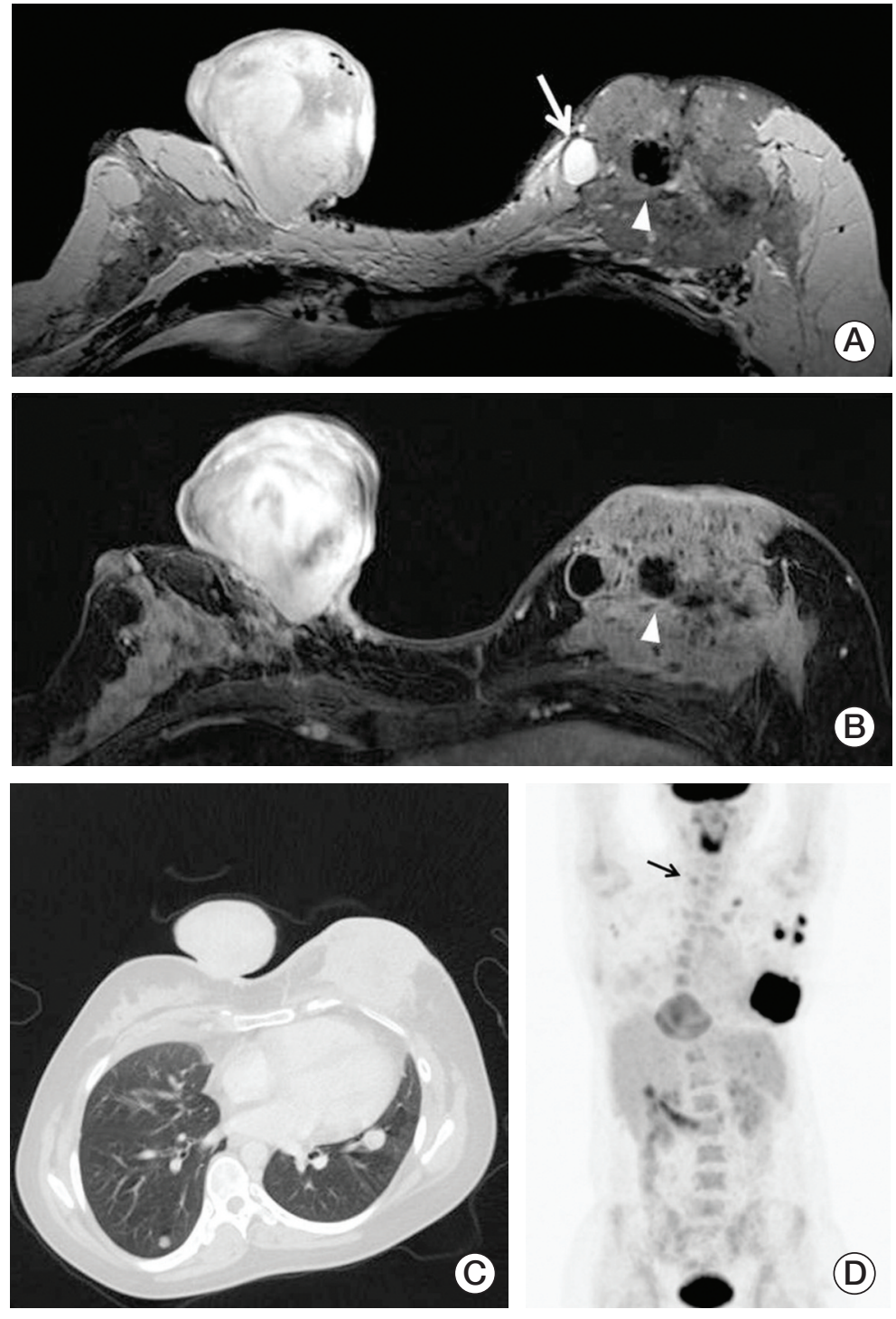

Fig. 1. A 6.8-cm-sized mass arising from right breast skin. The mass reveals $\mathrm{T} 2$ high signal (A) and intense enhancement after contrast enhancement (B). A 9.7-cm-sized, irregular shaped left breast mass involving skin and chest wall muscle. Heterogeneous enhancement with internal cyst (arrow in A) and signal void due to macro-calcification (arrowhead in A and B) is noted. (C) Lung window image of the breast computed tomography shows well-defined, small round nodules in both lung fields. (D) Positron emission tomography image demonstrates two large masses with different fluorodeoxyglucose (FDG) avidity in the bilateral breast. Several lymph nodes with variable FDG uptake are noted in the bilateral axillary and left supraclavicular area. Small nodular uptake (arrow) is seen in the right thyroidal area. 

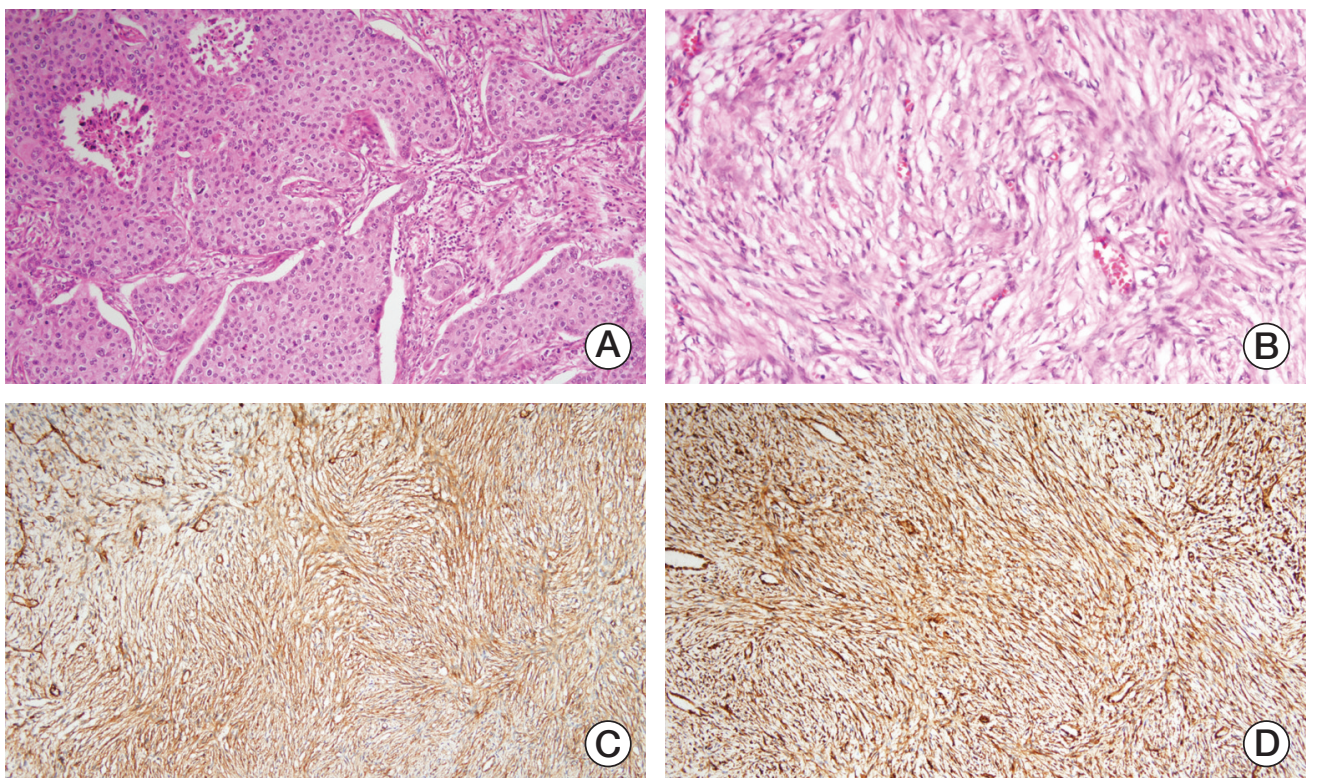

Fig. 2. (A) Diffuse infiltration of small carcinoma cells in the left breast (H\&E staining, $\times 100)$. (B) Poorly differentiated spindle cell tumor in the right breast (H\&E staining, $\times 200)$. Immunohistochemistry showed strong positive staining for CD34 (C) and vimentin (D) in the spindle cell component $(\times 100)$.

\section{Case Report}

A 29-year-old woman visited our hospital with a palpable left breast mass and right chest wall mass in March of 2017. She had some unique phenotypic features and medical history. Her height, weight, and head circumference were 167 $\mathrm{cm}, 64 \mathrm{~kg}$, and $58.1 \mathrm{~cm}$, respectively. The mean head circumference of average ( \pm standard deviation) adult women in Korea is $55.2 \pm 1.47 \mathrm{~cm} \mathrm{[4].} \mathrm{Her} \mathrm{mother} \mathrm{said} \mathrm{that} \mathrm{her} \mathrm{birth}$ weight was about $3.5 \mathrm{~kg}$ and she looked like she had a big head at birth. She was diagnosed with some developmental delay during infancy and childhood. She had undergone a near-total thyroidectomy for multinodular goiter at the ages of 9 and 14 years, respectively. The pathology report confirmed nodular hyperplasia. However, there is no family history of breast or other cancers. She has two sisters and they also have not had any special illnesses.

We conducted tests on the patient. Breast magnetic resonance imaging (MRI) showed an irregularly shaped, 9.7-cmsized mass in the left and a well circumscribed, 6.8 -cm-sized mass arising from the subcutaneous fat layer of the right (Fig. 1A and B). Multiple pulmonary nodules were detected on breast computed tomography (CT) (Fig. 1C). A core needle biopsy of both was performed and pathological examination revealed a poorly differentiated carcinoma in the left and poorly differentiated spindle cell tumor in the right.
${ }^{18} \mathrm{~F}$-fluorodeoxyglucose (FDG) positron emission tomography-CT showed an intensely FDG-avid mass (maximum standardized uptake value [SUV $\left.V_{\max }\right] 12.5$ ) in the left breast and a heterogeneously and moderately FDG-avid pedunculated subcutaneous mass $\left(\mathrm{SUV}_{\max } 4.6\right)$ in the right breast. There were multiple pulmonary nodules less than $1.5 \mathrm{~cm}$ in size with mild FDG uptake (SUV $\mathrm{Sax}_{\max } 1.7$ ) and focal nodular lesions with moderate FDG uptake in the right thyroid lobe (SUV $\max$ 3.4) (Fig. 1D).

She underwent a modified radical bilateral mastectomy in March 2017. Histopathological examination of the left breast mass revealed an invasive ductal carcinoma with squamous metaplastic carcinoma (Fig. 2A). Tumor size was $8 \mathrm{~cm}$ and there were 11 metastatic lymph nodes among the 31-dissected left axillary lymph nodes. Thus, pathological staging was pT3N3aMx. Immunohistochemical analyses showed negative staining for estrogen receptor, progesterone receptor, and human epidermal growth factor receptor 2. Histopathological examination of the right mass revealed a dermatofibrosarcoma protuberans, grade 2 (Fig. 2B-D). Tumor size was $7.5 \mathrm{~cm}$ diameter and there were no metastatic right axillary lymph nodes. Thus, pathological staging was pT2aNOMx.

In addition, we found that she had more phenotypic features of importance. She had mucosal papillomatous lesions on the upper gingiva and hard palate (Fig. 3A). There were no other mucocutaneous lesions such as trichilemmomas and 

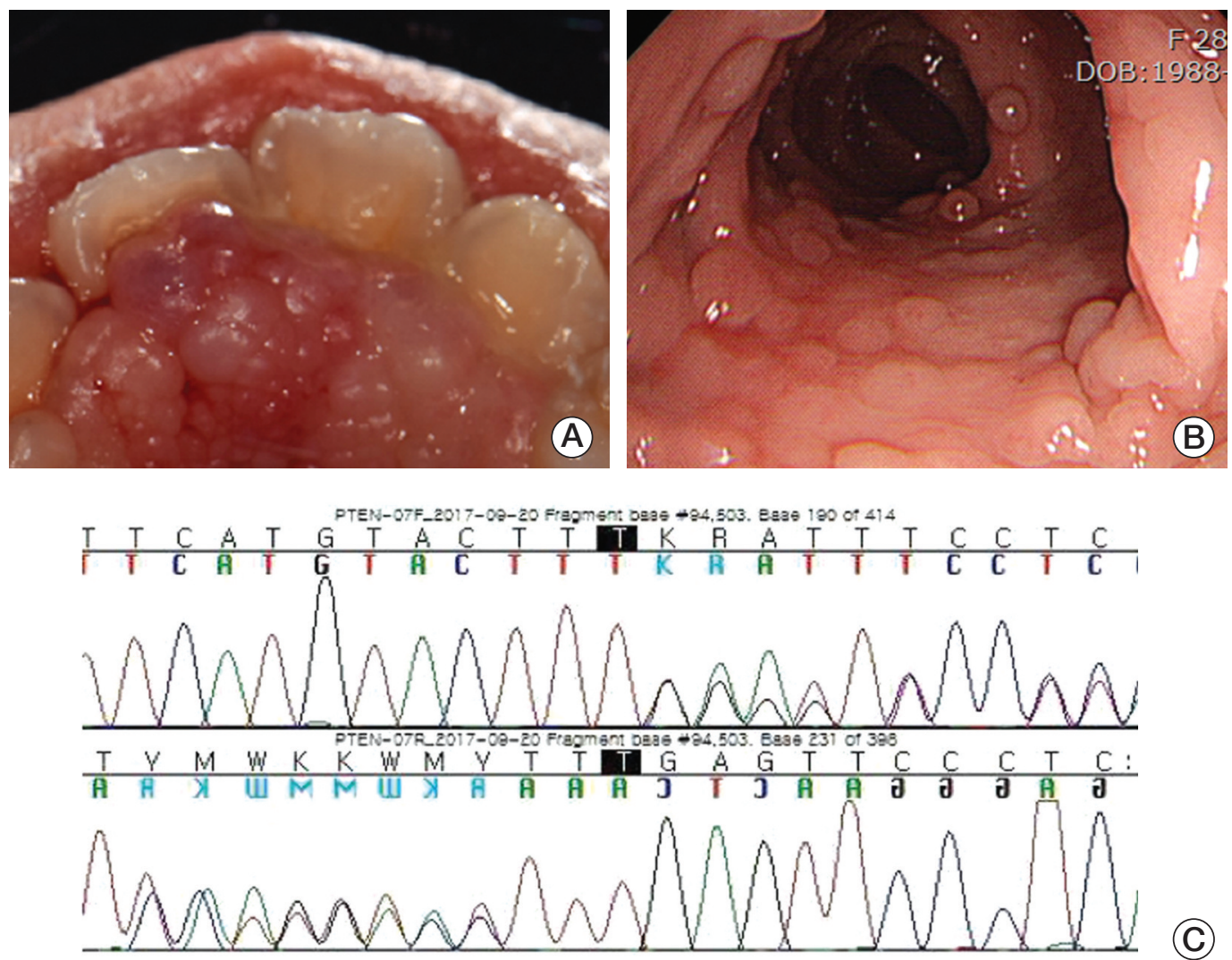

Fig. 3. (A) Mucosal papillomatous lesions on the upper gingiva and hard palate. (B) Multiple polys in the sigmoid colon. (C) Direct sequencing for whole exons including intron-exon boundarie of the PTEN gene. A frameshift PTEN mutation (c.723dupT [p.Glu242Terfs]) was identified in exon 7.

acral keratosis. X-ray images of her abdomen showed mild to moderated scoliosis. Gastroduodenoscopy and colonoscopy were performed and we found multiple gastrointestinal polyps distributed throughout the entire intestine (Fig. 3B). Based on her phenotypic features and medical history, we suspected that she might have PHTS. We performed a PTEN gene test and identified a frameshift PTEN mutation: c.723dupT (p.Glu242Terfs) in exon 7 (Fig. 3C). This is a pathogenic mutation of PTEN that was described previously in patients with PHTS [3,5].

After discussing her case among our multidisciplinary team, we decided to follow up on her pulmonary nodules while performing chemotherapy for advanced breast cancer because they were too small to make a diagnosis by CT-guided percutaneous lung biopsy. She received four cycles of adriamycin and cyclophosphamide. After the four cycles of chemotherapy, chest CT showed no change in pulmonary nodules. To determine an appropriate plan for further treatment, we performed a video-assisted thoracoscopic wedge resection of pulmonary nodule. Surprisingly, histopatholog- ical examination revealed colloid-filled thyroid follicles with various sizes in the lung (Fig. 4A and B). Subsequently, a core needle biopsy of her right thyroid nodule was performed and revealed a follicular neoplasm. She received adjuvant weekly paclitaxel chemotherapy for advanced breast cancer. After the completion of 12 weekly cycles of paclitaxel, she underwent both total thyroidectomy with sentinel lymph node biopsy and pathologic report revealed minimally invasive follicular carcinoma (Fig. 4C and D). We plan to perform radioactive iodine therapy. Her family will receive genetic counseling from a specialist.

\section{Discussion}

The PTEN tumor suppressor gene on chromosome 10q23 codes for a phosphatase that antagonizes the phosphoinositol-3-kinase/AKT signaling pathway and suppresses cell 

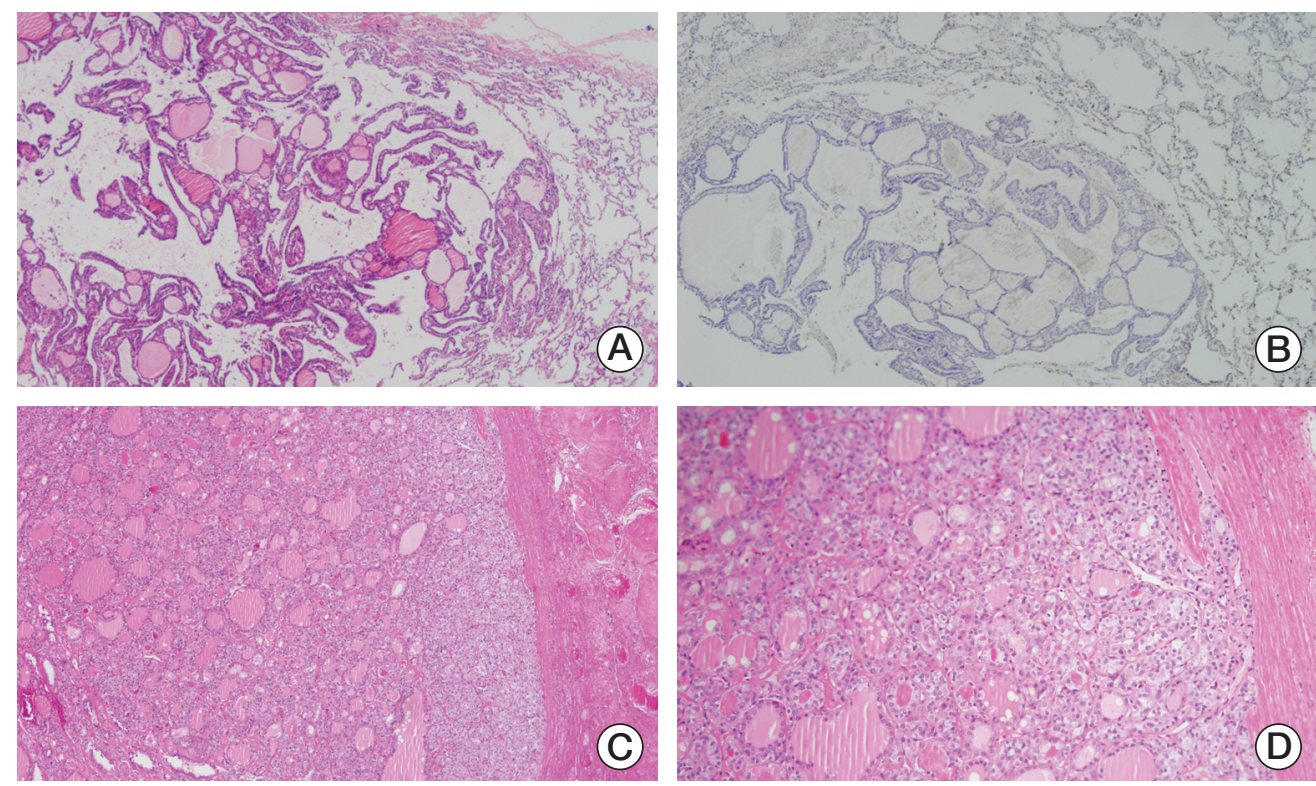

Fig. 4. (A) A well-circumscribed thyroid tissue including various sized-follicles in the lung parenchymal tissue (H\&E staining, $\times 100)$. (B) Negative immunohistochemical staining for galectin-3 $(\times 200)$. Capsular invasion in minimally invasive follicular carcinoma $(\times 40)(C)$ and $(\times 100)(D)$.

survival and cell proliferation [6]. CS and BRRS are the most commonly reported syndromes of PHTS, which is characterized by multiple hamartomas caused by germline mutations in PTEN. The clinical diagnostic criteria of CS established by the International Cowden Consortium are subdivided into three main categories: pathognomonic, major, and minor [1]. The international diagnostic criteria of BRRS have not been established yet, but the hallmark features of BRRS are defined as the presence of macrocephaly, lipomas, and hamartomatous intestinal polyposis, and in males, pigmented macules of the glans penis $[1,7]$. Other features described in BRRS include thyroid adenoma, lymphatic malformation, hemangiomas, high birth weight, proximal muscle myopathy, joint hyperextensibility, pectus excavatum, scoliosis, developmental delay, and intellectual deficiency [7]. Generally, most BRRS cases are reported with a childhood onset, often with delayed motor and intellectual development, while most CS cases are reported by dermatologists and oncologists in adults.

However, both conditions actually have many clinical features in common, and it is sometimes difficult to distinguish CS and BRRS clearly $[7,8]$. Our patient also had some features of both CS and BRRS. Her BRRS-related features include macrocephaly, delayed motor and intellectual development, hamartomatous intestinal polyposis, and thyroid hyperplasia in childhood. As features of CS, she has shown oral mucosal papillomatosis of pathognomonic criteria, breast cancer and macrocephaly as major criteria, and multinodular goiter and hamartomatous intestinal polyps as minor criteria. One of the operational diagnoses of CS includes two or more major criteria, of which one must be macrocephaly. In addition, more than $80 \%$ of individuals who met the international criteria of CS were found to have PTEN mutations, which have also been identified in approximately $60 \%$ of patients with BRRS $[1,2]$. Because of these clinically and genetically overlapping features, BRRS and CS are considered different phenotypical expressions of the same allelic syndrome. Some researchers have suggested that CS and BRRS represent one condition with variable expression and age-related penetrance [8]. Individuals with both CS and BRRS within the same family (CS/BRRS overlap families) and with phenotypes more consistent with CS or BRRS having the same PTEN mutations have also been reported previously $[3,5,7]$.

Despite the past thyroidectomy, our patient still had an enlarged thyroid gland. Multiple pulmonary nodules turned out to be thyroid tissues. Similarly, Cheng et al. [9]. reported a case of bilateral pulmonary thyroid nodules with a history of multinodular thyroid goiter. This is a very rare and interesting case. Treatment for thyroid and intrapulmonary thyroid tissue and continuous follow-up are needed.

Our patient has a frameshift PTEN mutation: c.723dupT (p.Glu242Terfs) in exon 7. Marsh et al. [3] previously reported that this mutation was identified in CS family. Bubien et al. [10] investigated the genotypic and phenotypic 
features of 146 patients with PHTS to define cancer risk according to the type and location of PTEN mutations. They found a patient with c.723dupT PTEN mutation who had macrocephaly, mucocutaneous lesion, and benign thyroid and breast disease, but no cancer [10]. However, our patient has experienced breast cancer and soft tissue sarcoma at a relatively young age.

Patients with CS are associated with an increased risk for developing breast, thyroid, endometrial, and renal cancers [1]. Bubien et al. [10] reported that overall $40 \%$ of 146 patients with PTEN mutations developed a cancer, with one quarter of these developing tumors across two or three different sites. Cancer was the main cause of death at a young age in patients with PHTS. Therefore, cancer surveillance is one of the most important strategies of disease management. Cancer screening recommendations have not been formally instituted for BRRS, PS, or PSL. However, previous studies reported that the cancer risk in patients with BRRS who harbor PTEN mutations was similar to that of individuals with CS [7]. Thus, all individuals with germline PTEN mutations should adopt the cancer surveillance strategies proposed for patients with CS [1]. Comprehensive physical examinations are recommended yearly. Patients aged younger than 18 years should be assessed for psychomotor development, dermatological and thyroid lesions, and vascular malformations. The assessment at diagnosis in adults includes dermatologi- cal and neurological examination, mammography and breast MRI, thyroid ultrasound, transvaginal ultrasound, upper gastrointestinal endoscopy, colonoscopy, and renal ultrasound $[1,10]$.

We can learn three important points while experiencing our case. First, PHTS has phenotypic heterogeneity and there are significant overlapping features between CS and BRRS. Thus, a collective diagnosis of PHTS may be better than a distinction between CS and BRRS. Second, our patient has been characterized by unique phenotypic features of PHTS since she was a child. However, genetic testing was performed after cancers were diagnosed in her adulthood. If clinicians meet children with developmental delay, macrocephaly, and mucocutaneous lesions, they should suspect PHTS and consider genetic testing. Third, unlike a case with a frameshift mutation of PTEN (c.723dupT) described previously [10], different types of cancer have developed in our present patient. Finally, we hope that our experience will help clinicians identify patients as appropriate candidates for PTEN testing in clinical practice. In addition, continuous studies of cancer surveillance, long-term follow-up, and prognosis in individuals with PHTS are needed.

\section{Conflicts of Interest}

Conflict of interest relevant to this article was not reported.

\section{References}

1. Hobert JA, Eng C. PTEN hamartoma tumor syndrome: an overview. Genet Med. 2009;11:687-94.

2. Pilarski R, Stephens JA, Noss R, Fisher JL, Prior TW. Predicting PTEN mutations: an evaluation of Cowden syndrome and Bannayan-Riley-Ruvalcaba syndrome clinical features. J Med Genet. 2011;48:505-12.

3. Marsh DJ, Coulon V, Lunetta KL, Rocca-Serra P, Dahia PL, Zheng Z, et al. Mutation spectrum and genotype-phenotype analyses in Cowden disease and Bannayan-Zonana syndrome, two hamartoma syndromes with germline PTEN mutation. Hum Mol Genet. 1998;7:507-15.

4. Bushby KM, Cole T, Matthews JN, Goodship JA. Centiles for adult head circumference. Arch Dis Child. 1992;67:1286-7.

5. Eng C. PTEN: one gene, many syndromes. Hum Mutat. 2003;22:183-98.

6. Yin Y, Shen WH. PTEN: a new guardian of the genome. Oncogene. 2008;27:5443-53.
7. Marsh DJ, Kum JB, Lunetta KL, Bennett MJ, Gorlin RJ, Ahmed $\mathrm{SF}$, et al. PTEN mutation spectrum and genotype-phenotype correlations in Bannayan-Riley-Ruvalcaba syndrome suggest a single entity with Cowden syndrome. Hum Mol Genet. 1999;8:1461-72.

8. Lachlan KL, Lucassen AM, Bunyan D, Temple IK. Cowden syndrome and Bannayan Riley Ruvalcaba syndrome represent one condition with variable expression and age-related penetrance: results of a clinical study of PTEN mutation carriers. J Med Genet. 2007;44:579-85.

9. Cheng H, Yang L, Xiong J, Peng J, Ruan Q. Multiple thyroid nodules in the lung: metastasis or ectopia? Diagn Pathol. 2015;10:61.

10. Bubien V, Bonnet F, Brouste V, Hoppe S, Barouk-Simonet E, David A, et al. High cumulative risks of cancer in patients with PTEN hamartoma tumour syndrome. J Med Genet. 2013;50: 255-63. 